\title{
The Aurora kinase inhibitor ZM447439 accelerates first meiosis in mouse oocytes by overriding the spindle assembly checkpoint
}

\author{
Simon I R Lane, Heng-Yu Chang, Phoebe C Jennings and Keith T Jones \\ School of Biomedical Sciences, University of Newcastle, Callaghan, New South Wales 2308, Australia \\ Correspondence should be addressed to KT Jones; Email: keith.jones@newcastle.edu.au
}

\begin{abstract}
Previous studies have established that when maturing mouse oocytes are continuously incubated with the Aurora inhibitor ZM447439, meiotic maturation is blocked. In this study, we observe that by altering the time of addition of the inhibitor, oocyte maturation can actually be accelerated by $1 \mathrm{~h}$ as measured by the timing of polar body extrusion. ZM447439 also had the ability to overcome a spindle assembly checkpoint (SAC) arrest caused by nocodazole and so rescue polar body extrusion. Consistent with the ability of the SAC to inhibit cyclin B1 degradation by blocking activation of the anaphase-promoting complex, we could also observe a rescue in cyclin B1 degradation when ZM447439 was added to nocodazole-treated oocytes. The acceleration of the first meiotic division by ZM447439, which has not been achieved previously, and its effects on the SAC are all consistent with the proposed mitotic role of Aurora B in activating the SAC. We hypothesize that Aurora kinase activity controls the SAC in meiosis I, despite differences to the mitotic cell cycle division in spindle architecture brought about by the meiotic mono-orientation of sister kinetochores.

Reproduction (2010) 140 521-530
\end{abstract}

\section{Introduction}

There has been much interest in what controls the first meiotic division (meiosis I) in oocytes because it is particularly error prone in humans, resulting in homolog non-disjunction such as trisomy 21 (Hunt \& Hassold 2008, Jones 2008). It is also a unique type of chromosome division, where sister kinetochores adopt a side-by-side configuration and consequently are mono-orientated. In meiosis II, and in all other mitotic divisions, sister kinetochores adopt a back-to-back configuration and consequently are bi-orientated (Brar \& Amon 2008, Holt \& Jones 2009). Such a configuration allows homologous chromosomes to divide reductionally in meiosis I but sister chromatids to divide equationally in meiosis II and mitosis. A further facet of meiosis I is an extremely protracted prometaphase, lasting several hours, during which the spindle assembles for the most part without kinetochore-microtubule attachments (Brunet et al. 1999).

In the mitotic cell cycle division, the anaphasepromoting complex/cyclosome (APC) is essential for segregating chromosomes, and its activity is tightly controlled to prevent aneuploidy (Acquaviva \& Pines 2006, Peters 2006). Its ubiquitin ligase activity leads, through the $26 \mathrm{~S}$ proteasome, to degradation of cyclin B1, so reducing CDK1 activity and also to degradation of securin, so freeing separase. The spindle assembly checkpoint (SAC) inhibits the APC during prometaphase and in so doing prevents aneuploidy (Musacchio \& Salmon 2007). Thus, the loss of SAC function in mitosis causes premature APC activity and consequently segregation of sister chromatids before full chromosome congression and bi-orientation, leading to aneuploidy in daughter cells and tumorigenesis (Li et al. 2009a).

APC-mediated cyclin B1 and securin degradation are also essential for the disjunction of homologs in maturing mammalian oocytes (Herbert et al. 2003, Terret et al. 2003, Reis et al. 2007). In one very interesting study using the $\mathrm{XO}$ mouse, the univalent $X$ chromosome was observed to divide reductionally in oocytes without any block to anaphase (LeMaire-Adkins et al. 1997). Consequently, it was concluded that the SAC was absent from female meiosis I. However, more detailed examination of Mad and Bub proteins, which are members of the SAC, has shown that SAC activation is an essential feature of this reductional division. Thus, knock out, knock down, or inhibition of Mad and Bub family members all lead to aneuploidy as a consequence of premature APC activation (Terret et al. 2003, Tsurumi etal. 2004, Homer et al. 2005, 2009, Yin et al. 2006, Niault et al. 2007, Li et al. 2009b, McGuinness et al. 2009).

The high incidence of aneuploidy resulting from mis-segregation of homologs in meiosis I errors is therefore unlikely to be due simply to an absence of the SAC. Nevertheless, the observations on the oocytes of the XO mouse, as well as other studies demonstrating high rates of aneuploidy in the absence of SAC activation 
(Reis et al. 2007), suggest that the SAC in oocytes may not respond to the same cues as it does in mitotically dividing cells and they may be insensitive or leaky in response to some arrangements of kinetochore-microtubule attachments during meiosis I. Further investigations into how the SAC is controlled and what it responds to in oocytes are therefore worthwhile pursuits.

With respect to the mitotic cell cycle division, in recent years, it has been established that the SAC is switched on through Aurora B kinase, which is a member of the chromosomal passenger complex (Kallio et al. 2002, Ditchfield et al. 2003, Hauf et al. 2003, Dewar et al. 2004, Lan et al. 2004, Kelly \& Funabiki 2009, Liu et al. 2009). Aurora B destabilizes incorrect microtubule-kinetochore attachments, and is switched off only when tension across the sister kinetochores, generated by bi-orientation, physically stretches centromeric Aurora B away from the kinetochore (Liu et al. 2009). The small molecule inhibitor, ZM447439, has proved useful to study Aurora B kinase activity independent of any structural function within the chromosomal passenger complex (Ditchfield et al. 2003). However, ZM447439 is better regarded as a general Aurora inhibitor because it has a similar $\mathrm{IC}_{50}$ for both Auroras A and B (Ditchfield et al. 2003); and is also likely to inhibit Aurora $C$, which has sequence homology to Aurora B (Tseng et al. 1998) and can rescue cells from loss of Aurora B (Slattery et al. 2009). Aurora C is likely to be relevant to meiosis since one recent study has shown that it is the most predominant Aurora kinase in oocytes
(Yang et al. 2010). If Aurora kinases function in meiosis as in somatic cell mitosis (Ditchfield et al. 2003, Hauf et al. 2003, Nilsson et al. 2008), ZM447439 should accelerate the passage of oocytes through meiosis I due to an SAC override, generate lagging chromosomes, and raise the incidence of aneuploidy in mature eggs. It should also overcome a SAC-imposed arrest, which could be measured directly in the form of increased APC activity. Although ZM447439 has been used previously on mouse oocytes, none of these observations have been made and instead its main effect is to block polar body extrusion, hence arresting rather than accelerating any passage through meiosis I (Swain et al. 2008, Shuda et al. 2009, Vogt et al. 2009). In this study, we have attempted a further examination of the effects of ZM447439 on mouse oocytes in order to determine whether we could uncover drug effects that would be consistent with similar studies in mitosis.

\section{Results}

\section{Early ZM447439 addition causes meiotic failure}

Immature dictyate-stage oocytes were cultured with $10 \mu \mathrm{M}$ ZM447439 45 min after milrinone washout, at which time about half of oocytes had undergone nuclear envelope breakdown (NEB; Fig. 1A). Time-lapse imaging was used to assess when polar body extrusion had occurred. In controls, the majority of oocytes $(94 \%$, $n=68$ ) extruded their polar bodies, at a time of $8.3 \pm 0.7 \mathrm{~h}$ after NEB (Fig. 1B), but no oocytes treated with
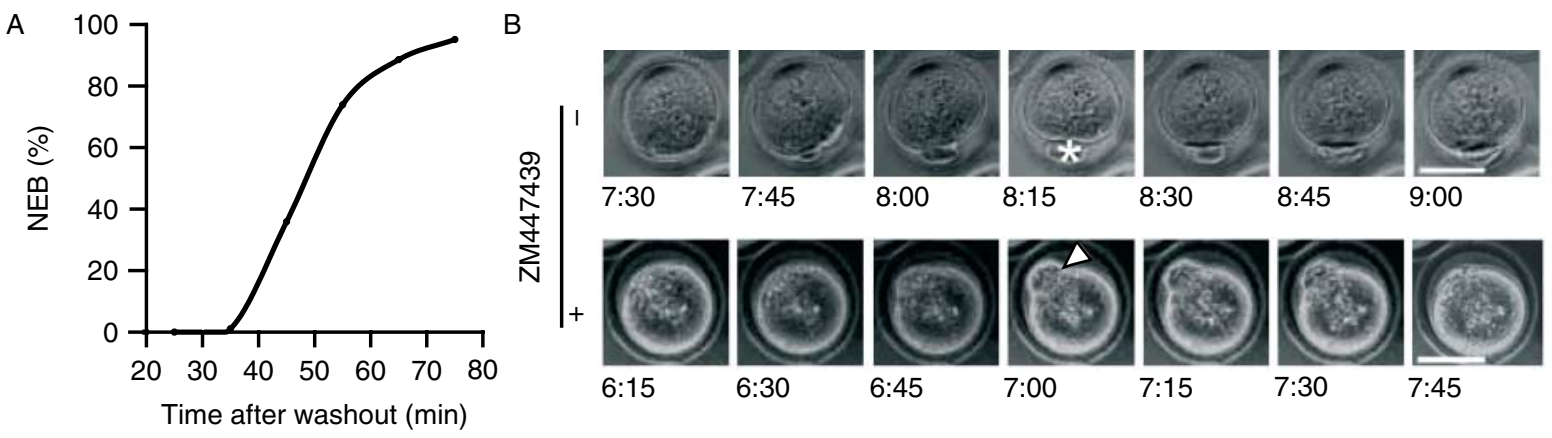

C
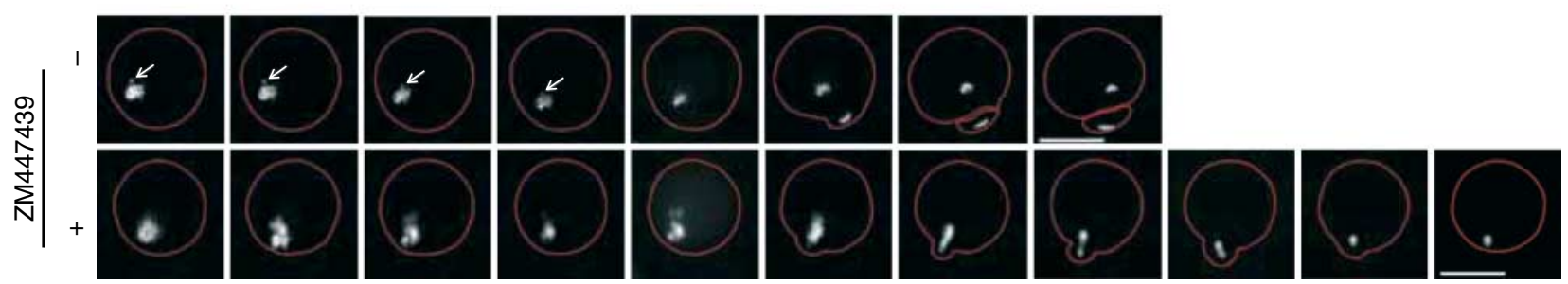

Figure 1 Continuous ZM447439 exposure blocks meiosis I. (A) Timing of nuclear envelope breakdown after milrinone washout. Cumulative percentage of oocytes undergoing NEB after washout from $1 \mu \mathrm{M}$ milrinone $(n=184)$. (B) Bright field time lapse of oocytes matured in media with ( $n=64$ ) or without ZM447439 ( $n=69$ ). Polar body extrusion was evident in control oocytes at the time indicated (asterisk), but was aborted in oocytes cultured in ZM447439 (arrowhead). Times are hours:minutes after NEB. (C) Images of chromatin (Hoechst) in live oocytes cultured with ( $n=13)$ or without $(n=31)$ ZM447439 during the period of permanent or aborted polar body extrusion. The red trace shows the location of the plasma membrane. Images are $15 \mathrm{~min}$ apart. Scale bars are $50 \mu \mathrm{m}$. 
ZM447439 extruded their polar bodies $(n=64)$. These observations on ZM447439 are consistent with previous studies that showed at doses between 1.5 and $20 \mu \mathrm{M}$, it inhibits polar body extrusion (Swain et al. 2008, Shuda et al. 2009, Vogt et al. 2009).

However, time-lapse imaging revealed oocytes did attempt extrusion of a polar body, albeit incomplete, observed as a protrusion in the plasma membrane, which lasted about $30 \mathrm{~min}$ before receding (Fig. 1B). The timing of this protrusion was earlier than controls at $7.3 \pm 1.5 \mathrm{~h}(P=0.001)$ after NEB. To explore this further, we combined bright field and chromosome (Hoechst) imaging to assess for congression and segregation errors during meiosis I. In control oocytes, chromosome congression led to the formation of a metaphase I plate, anaphase onset occurred after migration of the spindle to the cortex, and this resulted in the archetypical asymmetric division of an oocyte (Fig. 1C; $n=31$; Maro \& Verlhac 2002). During these divisions, no lagging chromosomes or other segregation defects were observed, and anaphase onset occurred shortly after the last homologs were aligned on the metaphase plate (Fig. 1C arrows). However, the initial defect in ZM447439 was a failure of homologs to congress. Instead, mal-aligned chromosomes migrated to the oocyte cortex, became spread along the length of the spindle axis, and within $30 \mathrm{~min}$, the extrusion receded back into the oocyte (Fig. 1C). We failed to study these factors further at greater resolution in live oocytes because we found that the increase in illumination needed to image the process actually blocked anaphase (brightness $\propto 1 /$ magnification $^{2}$ ).
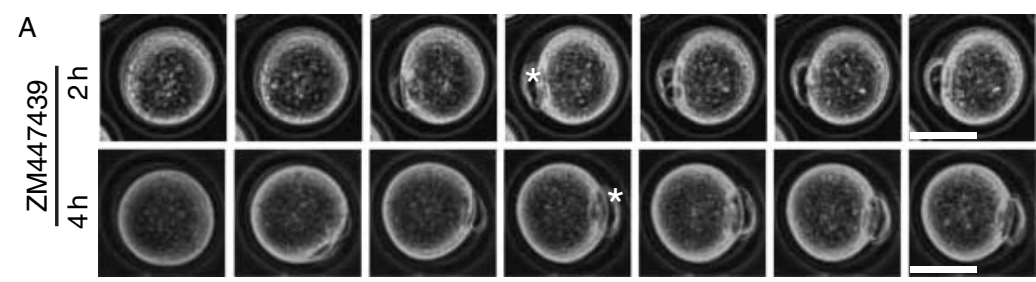

B

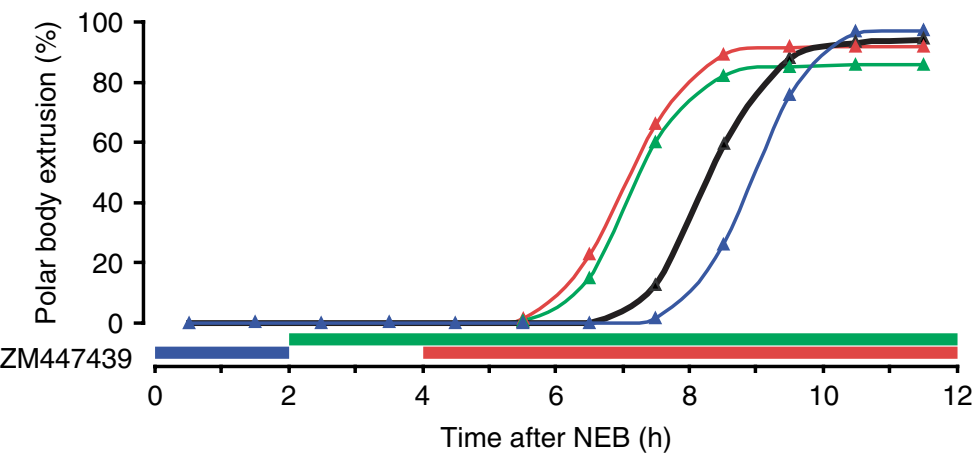

C

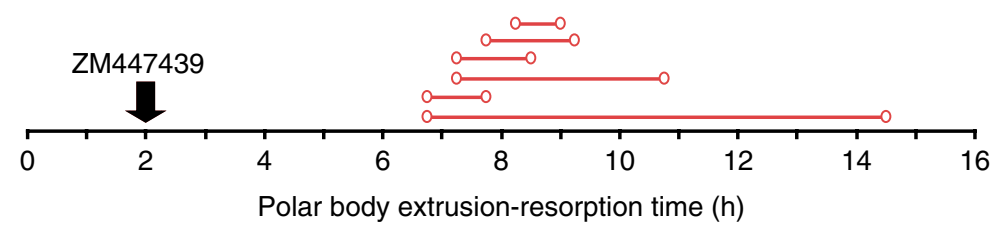

$\mathrm{D}$

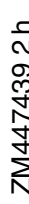

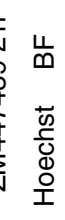
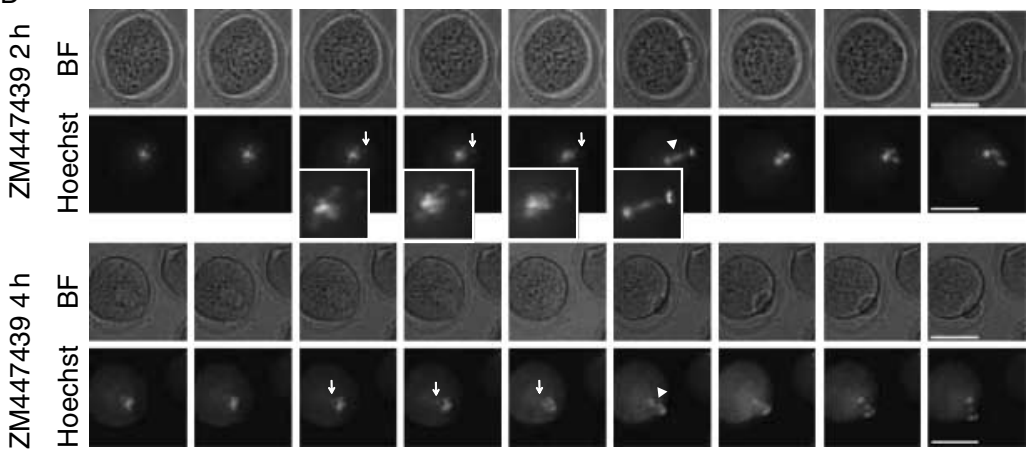
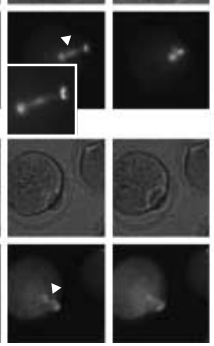

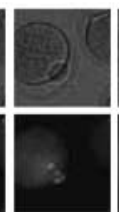

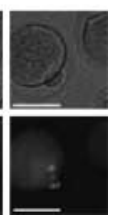

Figure 2 Prometaphase ZM447439 exposure accelerates meiosis I. (A) Bright field time lapse of oocytes matured in media with or without ZM447439 at the times indicated after NEB. These later ZM447439 additions led to morphologically normal polar bodies (asterisks). (B) Timing of polar body extrusion in oocytes $(n=56-99)$ incubated with ZM447439 for the times indicated. Addition of the Aurora kinase inhibitor during prometaphase I ( 2 and $4 \mathrm{~h}$ ) accelerated polar body extrusion. (C) In a very small number of oocytes $(n=6)$ treated with ZM447439 from $2 \mathrm{~h}$ after NEB, the polar bodies that were extruded were later resorbed. For those oocytes affected in this way, timelines are shown (red lines) indicating the period from polar body extrusion to later polar body resorption. Arrow indicates time of ZM447439 addition. (D) Bright field and Hoechst time-lapse images of live oocytes during the process of polar body extrusion after addition of ZM447439 at the times indicated ( $n=22)$, showing a failure to fully congress chromosomes during prometaphase (arrows) and lagging chromosomes during anaphase (arrowheads). Insets show enlargements of areas of interest. Scale bar is $50 \mu \mathrm{m}$. 


\section{Late ZM447439 addition causes meiotic acceleration}

We wondered whether the block to polar body extrusion would be conserved if $10 \mu \mathrm{M}$ ZM447439 was added later in oocyte maturation, 2 or $4 \mathrm{~h}$ after NEB. In fact, these later time points did not block polar body extrusion (Fig. 2A and B) but instead accelerated it by more than $1 \mathrm{~h}$ (Fig. $2 \mathrm{~B} ; P=0.001$ ). This acceleration was similar in timing to the attempted polar body extrusion when the inhibitor was added at $0 \mathrm{~h}$ (Fig. 1B). ZM447439 addition at $2 \mathrm{~h}$ resulted in some resorption of polar bodies, although at this later time, the rate was much lower that observed for aborted polar body at $0 \mathrm{~h}$ $(11 \%, n=57$ vs $75 \%, n=64$ at 0 h; Fig. $2 \mathrm{C})$. When the inhibitor was added at $4 \mathrm{~h}$, there was no decline in the polar body extrusion rate versus controls.

Given the opposing outcomes of adding ZM447439 at different times during oocyte maturation, inhibition versus acceleration of polar body extrusion, we wanted to determine whether the first $2 \mathrm{~h}$ after NEB represented a period of unique sensitivity to this drug. Therefore, oocytes were incubated in ZM447439 for $2 \mathrm{~h}$ after NEB and then washed into drug-free media. Although incubation with ZM447439 during the first $2 \mathrm{~h}$ of maturation caused a delay in the timing of extrusion of polar bodies ( $9.0 \pm 0.6 \mathrm{~h}$; Fig. 2B, blue), it did not block polar body extrusion to any extent (Fig. 2B; $97 \%, n=56$ ). Therefore, there appears no critical process during the first $2 \mathrm{~h}$ of meiotic resumption that cannot be completed after inhibitor washout. We conclude that the lack of chromosome segregation after $\mathrm{O} \mathrm{h}$ inhibitor addition was likely the primary reason for the block to polar body formation because chromatin in the region of the cleavage furrow has been shown in mitotic studies to be a very potent inhibitor of cytokinesis (Weaver et al. 2006, Steigemann et al. 2009).

We thought it important to examine how chromosomes segregated during meiosis I in oocytes cultured with the inhibitor at these 2 and $4 \mathrm{~h}$ time points. Timelapse imaging combined with Hoechst staining of chromatin revealed that there was often a failure to achieve homolog congression, with chromatin visible that was never at the spindle equator before anaphase ( $n=13 / 20$; Fig. 2D). Segregation of chromosomes was observed, but unlike controls (Fig. 1C) were often associated with lagging chromosomes or chromatin bridges $(n=15 / 20$; Fig. 2D). However, in contrast to the $0 \mathrm{~h}$ time point, chromosome segregation was associated with cytokinesis and the development of a cleavage furrow resulting in a polar body.

\section{ZM447439 overcomes a nocodazole-imposed SAC arrest}

The accelerated passage through meiosis I and the lagging chromosomes at anaphase, after ZM447439 addition at these later time points, would be consistent with its reported actions on mitotic cells, where it can be used to inhibit Aurora B, and so can overcome a SAC-imposed arrest. This acceleration of meiosis I complements findings in mouse oocytes where, by various nocodazole incubations, the period of meiosis I most sensitive to delay by nocodazole treatment was found to be 6-8 $\mathrm{h}$ after NEB (Brunet et al. 1999). In this study, we wanted to test whether ZM447439 would overcome a SAC arrest imposed by nocodazole.

Maturing oocytes at $4 \mathrm{~h}$ after NEB were cultured with nocodazole, a spindle poison that reduces microtubule stability. A dose of $400 \mathrm{nM}$ was used, the minimal dose needed to block polar body extrusion in the majority of oocytes (Wassmann et al. 2003). Time lapse of treated oocytes showed that polar body extrusion was very much inhibited $(24 \%, n=64$ vs $94 \%, n=68$ in vehicle controls; Fig. 3A) and those that did extrude polar bodies did so with greatly delayed timing $(11.9 \pm 1.5 \mathrm{~h}$; Fig. 3B). Addition of ZM447439 with nocodazole, however, caused a marked rescue of both the rate of polar body extrusion and the timing at which it occurred (55\%, $8.9 \pm 1.1 \mathrm{~h}$; Fig. $3 \mathrm{~A}$ and $\mathrm{B}$ ).

In nocodazole and ZM447439-treated oocytes that had extruded a polar body, chromatin was observed in both the oocyte and polar body (Fig. 4A). However, because of the presence of nocodazole and ZM447439, tubulin staining was weak and sister chromatids were not aligned on what would be expected to be the second meiotic metaphase spindle equator. A morphologically normal metaphase II spindle could be observed, however, if both drugs were washed out after polar body extrusion at $10 \mathrm{~h}$ (Fig. 4A). A similar disruption in the formation of a metaphase II spindle was seen when oocytes, matured in ZM447439 alone, were examined
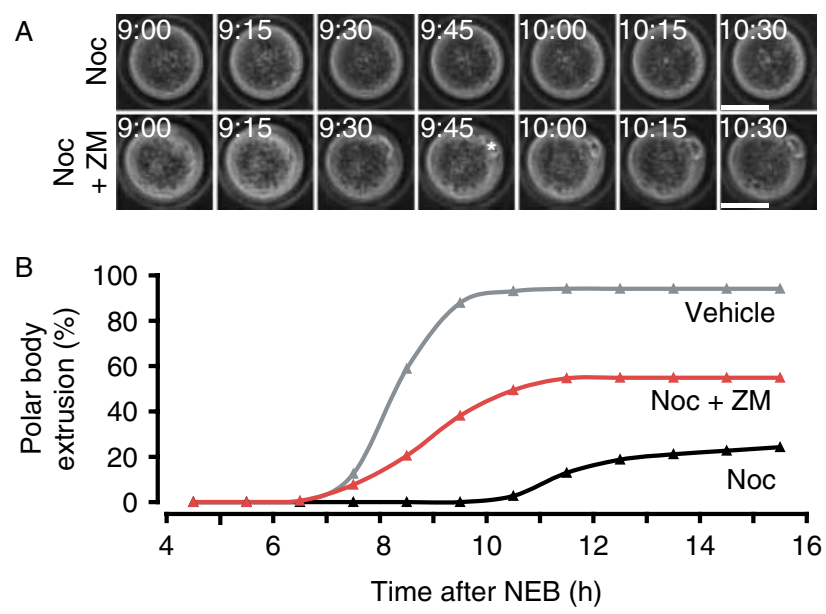

Figure 3 ZM447479 overcomes spindle checkpoint arrest in meiosis I. (A) Bright field time lapse of maturing oocytes cultured in nocodazole with or without ZM447439. Polar body extrusion was blocked by nocodazole but could be rescued (asterisks) by the Aurora kinase inhibitor. (B) Polar body extrusion rates in oocytes cultured in nocodazole $(n=64)$, nocodazole and ZM447439 $(n=83)$, or vehicle alone $(n=69)$. Scale bars are $20 \mu \mathrm{m}$. 
A
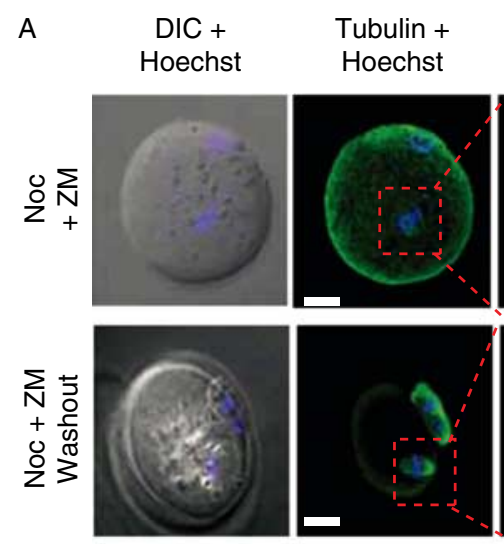
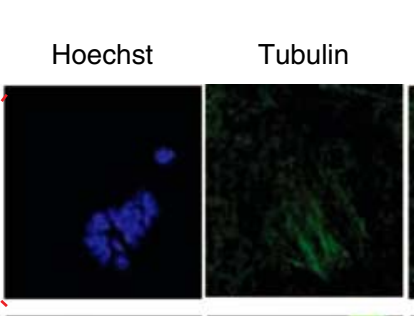

Tubulin + Hoechst
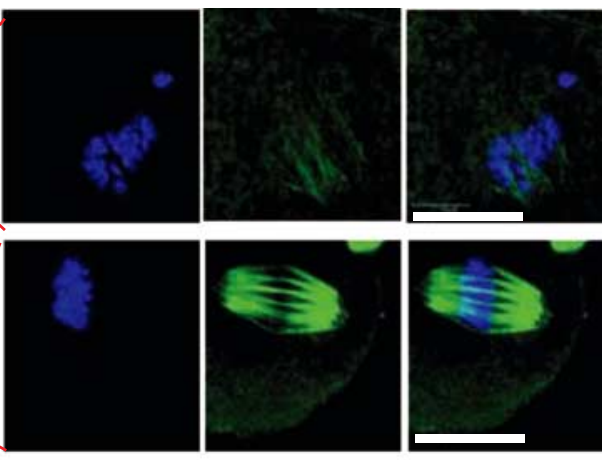

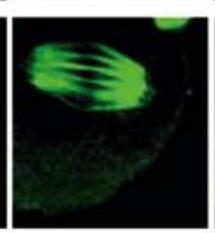

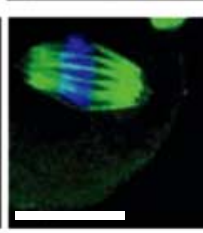

$\mathrm{DIC}+$ Hoechst
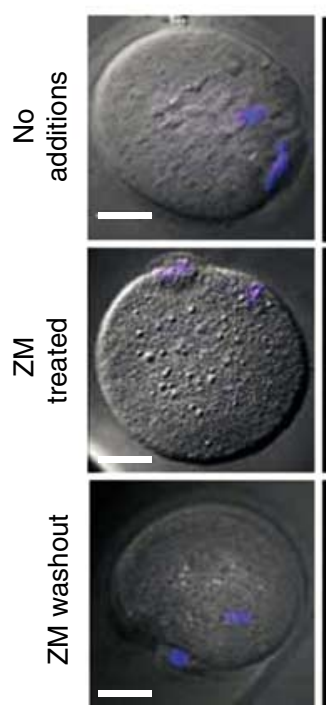

Hoechst
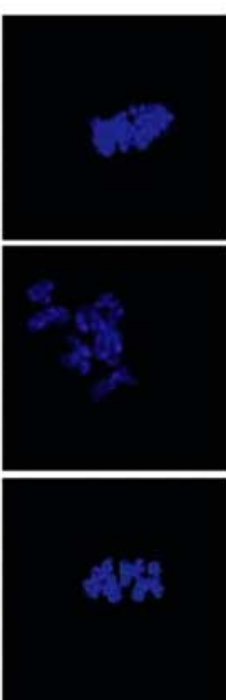

Tubulin
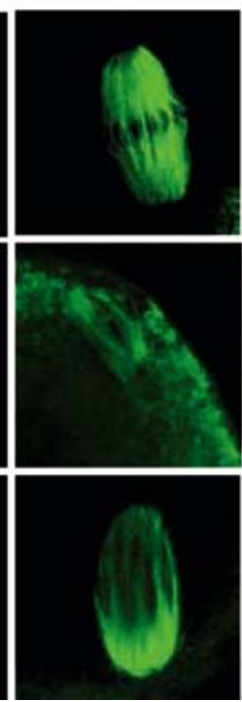

Tubulin + Hoechst

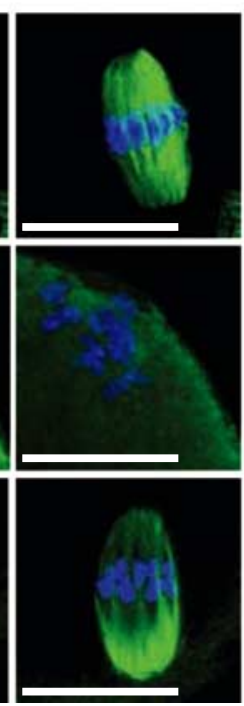

Figure 4 Nocodazole and ZM447479 effects on metaphase II spindle formation. (A) Confocal images for DIC, tubulin, and Hoechst in oocytes at $16 \mathrm{~h}$ after NEB, which had been matured in nocodazole and ZM447439 as indicated, washout of both the agents was at $10 \mathrm{~h}$. The addition of ZM447479 to nocodazole-treated oocytes led to the segregation of chromosomes $(n=13)$. A fully formed metaphase II spindle was not observed unless nocodazole and ZM447479 were washed out $(n=20)$. (B) Oocytes were treated with or without ZM447439 and were fixed $16 \mathrm{~h}$ after NEB, and then stained for tubulin and chromatin. Treatment with ZM447439 resulted in a weakly stained spindle and a lack of congression. Washout of ZM447439 at $10 \mathrm{~h}$ after NEB in oocytes that had extruded a polar body recovered a morphologically normal metaphase II spindle with bi-oriented chromosomes congressed at the spindle equator. Scale bar $20 \mu \mathrm{m}$. after polar body extrusion (Fig. 4B). A normal metaphase II spindle was only observed when mature oocytes were washed free of the inhibitor. We surmise that the activity of an Aurora kinase, likely to be Aurora B and/or $\mathrm{C}$, is therefore needed to assemble a bi-oriented metaphase II spindle.

\section{ZM447439 leads to cyclin B1 degradation in the presence of nocodazole}

APC activation and consequent cyclin B1 degradation are required for polar body extrusion in mouse oocytes (Ledan et al. 2001, Herbert et al. 2003, Hyslop et al. 2004, Reis et al. 2007). Therefore, release from a SAC-imposed meiotic arrest by ZM447439 should lead to cyclin B1 loss. To demonstrate this directly, oocytes were microinjected with cyclin B1-Venus cRNA and imaged for Venus fluorescent protein during maturation. As described previously (Herbert et al. 2003, Reis et al. 2007), in untreated oocytes, cyclin B1 levels gradually declined during oocyte maturation, reaching a minimum at the time of first polar body extrusion (Fig. 5). Addition of nocodazole stopped cyclin B1 degradation. However, degradation could be re-initiated when ZM447439 was added, demonstrating that direct release of the SAC by Aurora inhibition, probably Aurora B and/or C, had led to APC activation (Fig. 5).

\section{Aneuploidy associated with ZM447439 addition}

All the above data taken together suggest that ZM447439 leads to an acceleration in passage through meiosis I by overcoming the SAC-imposed delay to homolog disjunction; be it a nocodazole-induced delay or delay due to normal prometaphase SAC activity. One consequence of the accelerated passage though meiosis is that oocytes should show some increase in aneuploidy rates (Jones 2008). This is implied by the observation of lagging chromosomes and anaphase bridges in oocytes incubated with ZM447439. Therefore, oocytes were incubated with or without ZM447439 from $4 \mathrm{~h}$ after NEB. At $10 \mathrm{~h}$, oocytes that had extruded a polar body were assessed 


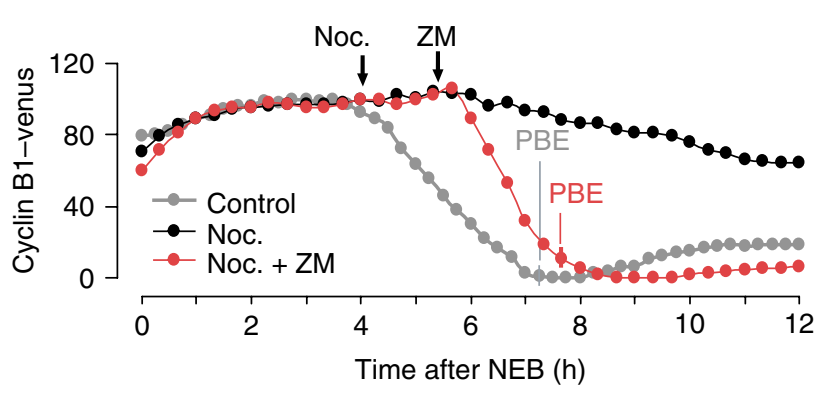

Figure 5 ZM447479 induces immediate cyclin B1 loss during a checkpoint arrest. Cyclin B1-Venus fluorescence in maturing oocytes injected with cRNA during G2 arrest. Nocodazole and ZM447439 were added at the points indicated. The loss in cyclin B1 fluorescence associated with oocyte maturation could be blocked by nocodazole addition but rescued by further addition of ZM447439. Traces are representative of 35 controls, 50 nocodazole, and 30 nocodazole +ZM447439 oocytes, each from at least two experiments.

by chromosome counts using a monastrol-based in situ spreading technique (Fig. 6A; Duncan et al. 2009). Low rates of aneuploidy, at consistent levels to other studies, were observed in the absence of ZM447439 (Reis et al. 2007, Duncan et al. 2009), but oocytes treated with the inhibitor, however, had a significantly higher, $15 \%$, aneuploidy rate (Fig. 6B; $P=0.004$, Fisher's exact test).

\section{Discussion}

There have been three previous studies on mouse oocytes examining the effects of the Aurora inhibitor ZM447439 on meiosis I, using a dose range of 1.5-20 $\mu \mathrm{M}$ (Swain et al. 2008, Shuda et al. 2009, Vogt et al. 2009). Independent of the dose used, the same observation was made in those studies as that made in this study: early ZM447439 addition to oocytes at the time of NEB is associated with a failure of maturing oocytes to complete meiosis I, morphologically evident by the lack of a polar body. Although this is now a consistent finding, it is nonetheless a somewhat disappointing observation, given that the expectation was to observe an acceleration of polar body extrusion. This expectation is based on the acceleration of meiosis I caused by loss of the SAC in oocytes, and the ability of ZM-induced Aurora B inhibition to inhibit the SAC in mitosis.

Investigations using Aurora inhibitors in oocytes of other species have produced differing results. For example, porcine oocyte incubation in ZM447439 results in prophase arrest (Jelinkova \& Kubelka 2006). This may be species specific because, similar to mouse oocytes, in bovine oocytes or clam oocytes, VX680 and ZM447439 respectively block meiosis in $\mathrm{MI}$ or in interkinesis (George et al. 2006, Uzbekova et al. 2008). Gadea \& Ruderman (2005) demonstrated in cycling Xenopus egg extracts that ZM447439 incubation disrupts spindle formation and chromosome condensation but does not arrest the cell cycle. Likewise, in mitotic cell lines, spindle and chromosome defects are observed but there is no block to cell cycle progression (Ditchfield et al. 2003). In the present study, we wished to address the role of Auroras in the SAC response of mouse oocytes. For this reason, the design of this study was adjusted to avoid blocking oocytes in MI. We found that an entirely different response to ZM447439 is uncovered if it is not added at NEB, but a few hours later. With such a design, ZM447439 did not block polar body extrusion, rather it accelerated it. This acceleration in itself suggests that the SAC is abrogated. Furthermore, the shortened meiosis I was associated with lagging chromosomes, anaphase bridges, and a rise in non-disjunction rates, all of which are suggestive of both an override of the SAC and a role for Auroras in ridding erroneous microtubulekinetochore attachments.

The 15\% non-disjunction rates achieved with ZM447439 were not as high as that for the 30\% rates achieved by Mad2 knockdown (Homer et al. 2005) but nonetheless represent a sixfold increase relative to controls. ZM447439 had the ability to overcome a meiotic arrest caused by nocodazole, a well-established SAC activator. We also demonstrated directly, through real-time loss in cyclin B1, release from SAC arrest when ZM447439 is added following nocodazole. In summary, the results help establish that Aurora kinase activity is important in activation of the SAC, and that the SAC is normally switched on during meiosis I to prevent premature homolog segregation and hence non-disjunction.

\section{ZM447439 inhibition of the Aurora family}

There are three members of the Aurora family, all with potential for being inhibited by ZM447439. ZM447439 has been shown directly to inhibit both Auroras A and B (Ditchfield et al. 2003), which have distinct cellular functions (Macurek et al. 2008, Seki et al. 2008). It is likely also to inhibit Aurora $C$, given this kinase evolved through gene duplication of Aurora B (Brown et al. 2004) and can rescue cells from Aurora B knockdown (Slattery et al. 2009). Aurora $C$ is predominantly expressed in the testis, where it has an essential and distinct role from Aurora B in late spermatogenesis (Kimmins et al. 2007). However, it is also found in many tissues at low levels (Lin et al. 2006) and has been shown to be present in oocytes. Indeed, all three Aurora transcripts are present in mouse oocytes (Swain et al. 2008, Shuda et al. 2009, Yang et al. 2010). Although it seems likely that Aurora C is present and functional in oocytes (Yang et al. 2010), there appear some contrasting studies on whether Aurora B is also present (Shuda et al. 2009, Vogt et al. 2009, Yang et al. 2010).

Aurora A knockdown arrests mouse oocytes in meiosis I (Solc et al. 2008), so it is possible that the addition of ZM447439 to oocytes from NEB onwards is preventing 
A

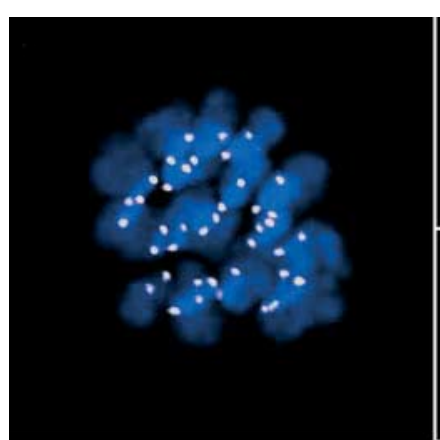

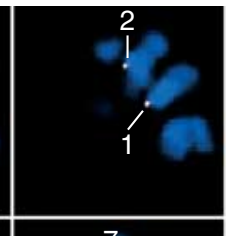
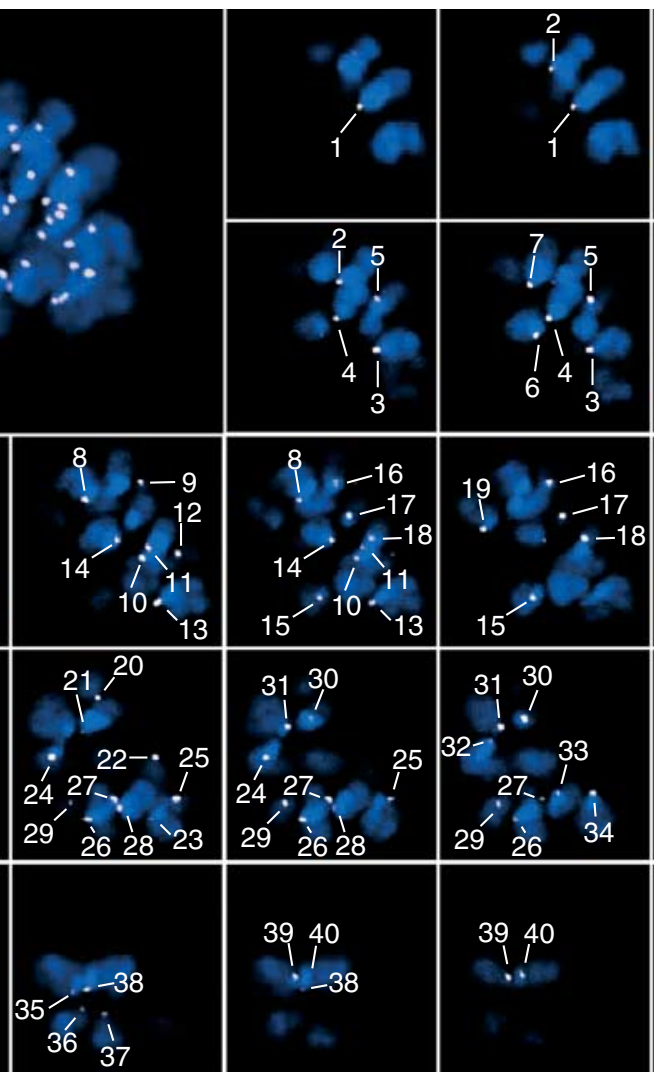

B
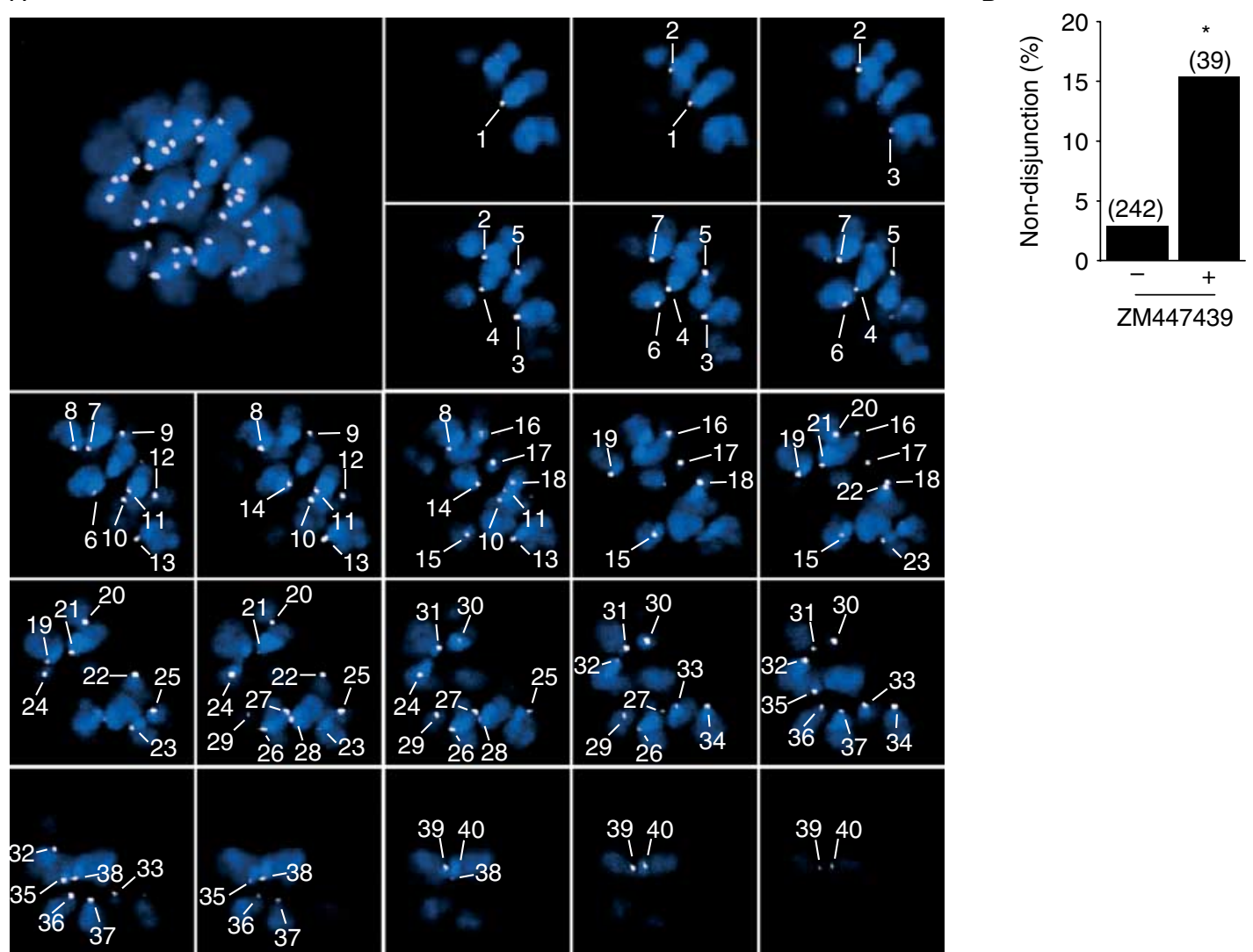

Figure 6 ZM447479 addition to oocytes during meiosis I results in aneuploidy. (A) The inset shows a flattened Z-stack series of images through a monastrol-treated oocyte; centromeres (CREST) are shown in white and chromatin (Hoechst) in blue. The following images show individual Z-planes from which the inset is comprised; 40 CREST-positive foci are counted in an euploid oocyte. (B) Percentage of non-disjunction rates in oocytes treated with or without ZM447439. Asterisk denotes a significant difference, $P=0.004$ (Fisher's test).

polar body extrusion through Aurora A kinase inhibition. Indeed, one of the major functions of Aurora $A$ is assembling the spindle (Carmena et al. 2009). It is, however, likely that the late ZM447439 addition, accelerating passage through meiosis I and overcoming SAC arrest, is through Aurora B or C inhibition, since Aurora A kinase is not involved in this process. Future studies are now needed to distinguish the roles of Auroras B and C in oocytes.

\section{The role of the SAC in oocytes}

Interest in the functionality and control of the SAC in oocytes stemmed from the observation that mouse oocytes possessing a univalent $X$ chromosome undergo meiotic maturation with relatively normal timing (LeMaire-Adkins et al. 1997). Assuming the SAC to be present in oocytes, one would have anticipated a cell arrest due to a failure in generating tension across the one remaining sister kinetochore pair, and indeed this is observed in the sex-reversed XO male mouse (Kot \& Handel 1990, Sutcliffe et al. 1991). It would be possible for the SAC to be satisfied if the $\mathrm{X}$ chromosome divided as it does in meiosis II; indeed, such equational divisions can occur in meiosis I when mice lack the synaptonemal complex component SYCP3 (Kouznetsova et al. 2007). However, with respect to the $\mathrm{XO}$ mouse, the majority of $X$ chromosomes segregate reductionally (LeMaireAdkins et al. 1997, LeMaire-Adkins \& Hunt 2000). So why did the lack of tension across the univalent not generate a robust meiotic arrest? It is not because the SAC is absent in oocytes. Many subsequent studies have reported on its presence and functionality in mouse oocytes (Terret et al. 2003, Homer et al. 2005, 2009, Yin et al. 2006, Niault et al. 2007, Hupalowska et al. 2008, Li et al. 2009b, McGuinness et al. 2009).

One possibility that would attempt to unify the data is that there are certain types of kinetochore-microtubule attachments that are poorly detected and corrected in oocytes. In addition, end-on microtubule-kinetochore attachments are not formed until very late in meiosis I (Brunet et al. 1999). Hence, there is an interest in this study and also in previous studies, in determining whether Auroras have any SAC role in the unusual first division of oocytes. During mitosis, Aurora B activity is thought to be important in correcting merotelic 


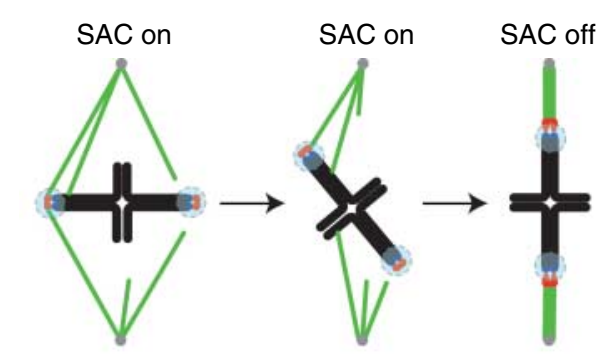

- Aurora B/C (centromere) Sister kinetochores

Aurora B/C activity

Figure 7 Model of Aurora and SAC activation in meiosis. Aurora B/C, located at the centromeres, will act to destabilize microtubulekinetochore interaction and so activate the SAC until the homologous chromosome pair is bi-oriented and under tension, removing centromeric Aurora away from kinetochore substrates.

attachments (Cimini et al. 2006, Rosasco-Nitcher et al. 2008), which are able to evade the SAC and results in aneuploidy (Cimini et al. 2001). Although it still remains possible that certain types of attachment are poorly detected in oocytes, the present observations suggest that Auroras can function in mouse meiosis in much the same way as they do in mitosis with respect to the SAC (Fig. 7). It is tempting to speculate that Aurora $C$ is a meiotic substitute for Aurora B, allowing oocytes to adapt to the unique challenges of meiosis $\mathrm{I}$, such as the monoorientation of sister kinetochores during meiosis, but that this substitution may also be the source of the leakiness observed in the meiotic SAC.

\section{Materials and Methods}

All chemicals were from Sigma-Aldrich unless stated otherwise. ZM447439 (10 mM; Tocris, Ellisville, MO, USA) and nocodazole $(400 \mu \mathrm{M})$ in DMSO were used at a dilution of $0.1 \%$.

\section{Oocyte collection}

Oocytes were collected from hormonally primed F1 hybrid mice (C57Bl6 females $\times$ CBA males) as described previously (Chang et al. 2009). Milrinone $(1 \mu \mathrm{M})$ was added to maintain arrest (Tsafriri et al. 1996). For long-term incubation, oocytes were cultured in MEM (Gibco) with $20 \% \mathrm{FCS}$ at $37^{\circ} \mathrm{C}$ in $5 \% \mathrm{CO}_{2}$.

\section{Microinjection and imaging}

Cyclin B1-Venus cRNA microinjection was performed as previously described (Reis et al. 2006). Epifluorescence images were captured using a Nikon Biostation IM fitted with a $\times 20$ objective. Images were analyzed using Metamorph software (Molecular Devices, Sunnyvale, CA, USA). Only oocytes that had undergone NEB within 20 min of the mean NEB time point were used in analysis.

\section{Immunofluorescence}

Oocytes were treated for $10 \mathrm{~h}$ in $200 \mu \mathrm{M}$ monastrol, then fixed in $4 \%$ formaldeyde, and permeabilized in Triton X-100 (Holt et al. 2010). Immunofluorescence was performed as described previously (Holt et al. 2010) using primary antibodies: antitubulin (1:400, Invitrogen) and CREST (1:400, Cortex Biochem, San Leandro, CA, USA) supplemented with $1 \%$ BSA and $0.2 \%$ Tween 20 overnight at $4{ }^{\circ} \mathrm{C}$. Secondary antibodies were Alexa 555- or 633-conjugated (Invitrogen). Oocytes were briefly stained with Hoechst $(20 \mu \mathrm{g} / \mathrm{ml})$ before mounting on glass slides with Citifluor (Citifluor Ltd, London, UK).

\section{Confocal imaging and aneuploidy analysis}

Confocal microscopy was performed using an Olympus FV1000 fitted with a $\times 60$ objective. Z-stacks were compiled with $0.5 \mu \mathrm{m}$ intervals. Analysis was performed with FV10-ASW 2.0 Viewer software (Olympus, Tokyo, Japan). Kinetochore counts were made by comparing CREST and Hoechst staining in each Z-plane through a stack of planes encompassing the entire spindle (Fig. 6A). All oocytes that were counted were made anonymous to eliminate the possibility of any observer bias, and confirmed by an independent second count.

\section{Statistical analysis}

Fisher's exact test and Student's t-test were performed using Minitab15 software (Minitab, State College, PA, USA).

\section{Figure preparation}

Figures were assembled using Adobe Illustrator CS4. Images shown in Fig. 1C were generated by creating an outline of the plasma membrane from bright field images using Adobe Photoshop CS4. The trace of the plasma membrane was then applied to the corresponding Hoechst image to create the overlay.

\section{Declaration of interest}

The authors declare that there is no conflict of interest that could be perceived as prejudicing the impartiality of the research reported.

\section{Funding}

This work was funded by the NHMRC, Australia (569202), to $\mathrm{K} T$ Jones. S I R Lane is the recipient of a Northcote graduate scholarship.

\section{References}

Acquaviva C \& Pines J 2006 The anaphase-promoting complex/cyclosome: APC/C. Journal of Cell Science 119 2401-2404. (doi:10.1242/jcs.02937)

Brar GA \& Amon A 2008 Emerging roles for centromeres in meiosis I chromosome segregation. Nature Reviews. Genetics 9 899-910. (doi:10. 1038/nrg2454) 
Brown JR, Koretke KK, Birkeland ML, Sanseau P \& Patrick DR 2004 Evolutionary relationships of Aurora kinases: implications for model organism studies and the development of anti-cancer drugs. BMC Evolutionary Biology 4 39. (doi:10.1186/1471-2148-4-39)

Brunet S, Maria AS, Guillaud P, Dujardin D, Kubiak JZ \& Maro B 1999 Kinetochore fibers are not involved in the formation of the first meiotic spindle in mouse oocytes, but control the exit from the first meiotic M phase. Journal of Cell Biology 146 1-12. (doi:10.1083/jcb.146.1.1)

Carmena M, Ruchaud S \& Earnshaw WC 2009 Making the Auroras glow: regulation of Aurora $\mathrm{A}$ and $\mathrm{B}$ kinase function by interacting proteins. Current Opinion in Cell Biology 21 796-805. (doi:10.1016/j.ceb.2009. 09.008)

Chang HY, Minahan K, Merriman JA \& Jones KT 2009 Calmodulindependent protein kinase gamma 3 (CamKIlgamma3) mediates the cell cycle resumption of metaphase II eggs in mouse. Development $\mathbf{1 3 6}$ 4077-4081. (doi:10.1242/dev.042143)

Cimini D, Howell B, Maddox P, Khodjakov A, Degrassi F \& Salmon ED 2001 Merotelic kinetochore orientation is a major mechanism of aneuploidy in mitotic mammalian tissue cells. Journal of Cell Biology 153 517-528. (doi:10.1083/jcb.153.3.517)

Cimini D, Wan X, Hirel CB \& Salmon ED 2006 Aurora kinase promotes turnover of kinetochore microtubules to reduce chromosome segregation errors. Current Biology 16 1711-1718. (doi:10.1016/j.cub.2006.07.022)

Dewar H, Tanaka K, Nasmyth K \& Tanaka TU 2004 Tension between two kinetochores suffices for their bi-orientation on the mitotic spindle. Nature 428 93-97. (doi:10.1038/nature02328)

Ditchfield C, Johnson VL, Tighe A, Ellston R, Haworth C, Johnson T, Mortlock A, Keen N \& Taylor SS 2003 Aurora B couples chromosome alignment with anaphase by targeting BubR1, Mad2, and Cenp-E to kinetochores. Journal of Cell Biology 161 267-280. (doi:10.1083/jcb. 200208091)

Duncan FE, Chiang T, Schultz RM \& Lampson MA 2009 Evidence that a defective spindle assembly checkpoint is not the primary cause of maternal age-associated aneuploidy in mouse eggs. Biology of Reproduction 81 768-776. (doi:10.1095/biolreprod.109.077909)

Gadea BB \& Ruderman JV 2005 Aurora kinase inhibitor ZM447439 blocks chromosome-induced spindle assembly, the completion of chromosome condensation, and the establishment of the spindle integrity checkpoint in Xenopus egg extracts. Molecular Biology of the Cell 16 1305-1318. (doi:10.1091/mbc.E04-10-0891)

George O, Johnston MA \& Shuster CB 2006 Aurora B kinase maintains chromatin organization during the MI to MII transition in surf clam oocytes. Cell Cycle 5 2648-2656.

Hauf S, Cole RW, LaTerra S, Zimmer C, Schnapp G, Walter R, Heckel A, van Meel J, Rieder CL \& Peters JM 2003 The small molecule Hesperadin reveals a role for Aurora B in correcting kinetochore-microtubule attachment and in maintaining the spindle assembly checkpoint. Journal of Cell Biology 161 281-294. (doi:10.1083/jcb.200208092)

Herbert M, Levasseur M, Homer H, Yallop K, Murdoch A \& McDougall A 2003 Homologue disjunction in mouse oocytes requires proteolysis of securin and cyclin B1. Nature Cell Biology 5 1023-1025. (doi:10.1038/ ncb1062)

Holt JE \& Jones KT 2009 Control of homologous chromosome division in the mammalian oocyte. Molecular Human Reproduction 15 139-147. (doi:10.1093/molehr/gap007)

Holt JE, Weaver J \& Jones KT 2010 Spatial regulation of APCCdh1-induced cyclin B1 degradation maintains G2 arrest in mouse oocytes. Development 137 1297-1304. (doi:10.1242/dev.047555)

Homer HA, McDougall A, Levasseur M, Yallop K, Murdoch AP \& Herbert M 2005 Mad2 prevents aneuploidy and premature proteolysis of cyclin B and securin during meiosis I in mouse oocytes. Genes and Development 19 202-207. (doi:10.1101/gad.328105)

Homer H, Gui L \& Carroll J 2009 A spindle assembly checkpoint protein functions in prophase I arrest and prometaphase progression. Science 326 991-994. (doi:10.1126/science.1175326)

Hunt PA \& Hassold TJ 2008 Human female meiosis: what makes a good egg go bad? Trends in Genetics 24 86-93. (doi:10.1016/j.tig.2007.11.010)

Hupalowska A, Kalaszczynska I, Hoffmann S, Tsurumi C, Kubiak JZ, Polanski Z \& Ciemerych MA 2008 Metaphase I arrest in LT/SV mouse oocytes involves the spindle assembly checkpoint. Biology of Reproduction 79 1102-1110. (doi:10.1095/biolreprod.108.069609)
Hyslop LA, Nixon VL, Levasseur M, Chapman F, Chiba K, McDougall A, Venables JP, Elliott DJ \& Jones KT $2004 \mathrm{Ca}(2+)$-promoted cyclin B1 degradation in mouse oocytes requires the establishment of a metaphase arrest. Developmental Biology 269 206-219. (doi:10.1016/j.ydbio. 2004.01.030)

Jelinkova L \& Kubelka M 2006 Neither Aurora B activity nor histone H3 phosphorylation is essential for chromosome condensation during meiotic maturation of porcine oocytes. Biology of Reproduction $\mathbf{7 4}$ 905-912. (doi:10.1095/biolreprod.105.047886)

Jones KT 2008 Meiosis in oocytes: predisposition to aneuploidy and its increased incidence with age. Human Reproduction Update $\mathbf{1 4}$ 143-158. (doi:10.1093/humupd/dmm043)

Kallio MJ, McCleland ML, Stukenberg PT \& Gorbsky GJ 2002 Inhibition of aurora B kinase blocks chromosome segregation, overrides the spindle checkpoint, and perturbs microtubule dynamics in mitosis. Current Biology 12 900-905. (doi:10.1016/S0960-9822(02)00887-4)

Kelly AE \& Funabiki H 2009 Correcting aberrant kinetochore microtubule attachments: an Aurora B-centric view. Current Opinion in Cell Biology 21 51-58. (doi:10.1016/j.ceb.2009.01.004)

Kimmins S, Crosio C, Kotaja N, Hirayama J, Monaco L, Hoog C, van Duin M, Gossen JA \& Sassone-Corsi P 2007 Differential functions of the Aurora-B and Aurora-C kinases in mammalian spermatogenesis. Molecular Endocrinology 21 726-739. (doi:10.1210/me.2006-0332)

Kot MC \& Handel MA 1990 Spermatogenesis in XO, Sxr mice: role of the $Y$ chromosome. Journal of Experimental Zoology 256 92-105. (doi:10. 1002/jez.1402560112)

Kouznetsova A, Lister L, Nordenskjold M, Herbert M \& Hoog C 2007 Bi-orientation of achiasmatic chromosomes in meiosis I oocytes contributes to aneuploidy in mice. Nature Genetics 39 966-968. (doi:10.1038/ ng2065)

Lan W, Zhang X, Kline-Smith SL, Rosasco SE, Barrett-Wilt GA, Shabanowitz J, Hunt DF, Walczak CE \& Stukenberg PT 2004 Aurora B phosphorylates centromeric MCAK and regulates its localization and microtubule depolymerization activity. Current Biology 14 273-286. (doi:10.1016/j.cub.2004.01.055)

Ledan E, Polanski Z, Terret ME \& Maro B 2001 Meiotic maturation of the mouse oocyte requires an equilibrium between cyclin B synthesis and degradation. Developmental Biology 232 400-413. (doi:10.1006/dbio. 2001.0188)

LeMaire-Adkins R \& Hunt PA 2000 Nonrandom segregation of the mouse univalent $X$ chromosome: evidence of spindle-mediated meiotic drive. Genetics 156 775-783.

LeMaire-Adkins R, Radke K \& Hunt PA 1997 Lack of checkpoint control at the metaphase/anaphase transition: a mechanism of meiotic nondisjunction in mammalian females. Journal of Cell Biology 139 1611-1619. (doi:10.1083/jcb.139.7.1611)

Li M, Fang X, Wei Z, York JP \& Zhang P 2009a Loss of spindle assembly checkpoint-mediated inhibition of Cdc20 promotes tumorigenesis in mice. Journal of Cell Biology 185 983-994. (doi:10.1083/jcb. 200904020)

Li M, Li S, Yuan J, Wang ZB, Sun SC, Schatten H \& Sun QY $2009 b$ Bub3 is a spindle assembly checkpoint protein regulating chromosome segregation during mouse oocyte meiosis. PLoS ONE 4 e7701. (doi:10.1371/ journal.pone.0007701)

Lin YS, Su LJ, Yu CT, Wong FH, Yeh HH, Chen SL, Wu JC, Lin WJ, Shiue YL, Liu HS et al. 2006 Gene expression profiles of the aurora family kinases. Gene Expression 13 15-26. (doi:10.3727/00000000 6783991962)

Liu D, Vader G, Vromans MJ, Lampson MA \& Lens SM 2009 Sensing chromosome bi-orientation by spatial separation of aurora B kinase from kinetochore substrates. Science 323 1350-1353. (doi:10.1126/science. 1167000)

Macurek L, Lindqvist A, Lim D, Lampson MA, Klompmaker R, Freire R, Clouin C, Taylor SS, Yaffe MB \& Medema RH 2008 Polo-like kinase- 1 is activated by aurora A to promote checkpoint recovery. Nature $\mathbf{4 5 5}$ 119-123. (doi:10.1038/nature07185)

Maro B \& Verlhac MH 2002 Polar body formation: new rules for asymmetric divisions. Nature Cell Biology 4 E281-E283. (doi:10.1038/ ncb1202-e281)

McGuinness BE, Anger M, Kouznetsova A, Gil-Bernabe AM, Helmhart W, Kudo NR, Wuensche A, Taylor S, Hoog C, Novak B et al. 2009 
Regulation of APC/C activity in oocytes by a Bub1-dependent spindle assembly checkpoint. Current Biology 19 369-380. (doi:10.1016/j.cub. 2009.01.064)

Musacchio A \& Salmon ED 2007 The spindle-assembly checkpoint in space and time. Nature Reviews. Molecular Cell Biology 8 379-393. (doi:10. 1038/nrm2163)

Niault T, Hached K, Sotillo R, Sorger PK, Maro B, Benezra R \& Wassmann K 2007 Changing Mad2 levels affects chromosome segregation and spindle assembly checkpoint control in female mouse meiosis I. PLOS ONE 2 e1165. (doi:10.1371/journal.pone.0001165)

Nilsson J, Yekezare M, Minshull J \& Pines J 2008 The APC/C maintains the spindle assembly checkpoint by targeting Cdc20 for destruction. Nature Cell Biology 10 1411-1420. (doi:10.1038/ncb1799)

Peters JM 2006 The anaphase promoting complex/cyclosome: a machine designed to destroy. Nature Reviews. Molecular Cell Biology 7 644-656. (doi:10.1038/nrm1988)

Reis A, Chang HY, Levasseur M \& Jones KT 2006 APCcdh1 activity in mouse oocytes prevents entry into the first meiotic division. Nature Cell Biology 8 539-540. (doi:10.1038/ncb1406)

Reis A, Madgwick S, Chang HY, Nabti I, Levasseur M \& Jones KT 2007 Prometaphase APCcdh1 activity prevents non-disjunction in mammalian oocytes. Nature Cell Biology 9 1192-1198. (doi:10.1038/ncb1640)

Rosasco-Nitcher SE, Lan W, Khorasanizadeh S \& Stukenberg PT 2008 Centromeric Aurora-B activation requires TD-60, microtubules, and substrate priming phosphorylation. Science 319 469-472. (doi:10.1126/ science.1148980)

Seki A, Coppinger JA, Jang CY, Yates JR \& Fang G 2008 Bora and the kinase Aurora a cooperatively activate the kinase Plk1 and control mitotic entry. Science 320 1655-1658. (doi:10.1126/science.1157425)

Shuda K, Schindler K, Ma J, Schultz RM \& Donovan PJ 2009 Aurora kinase $\mathrm{B}$ modulates chromosome alignment in mouse oocytes. Molecular Reproduction and Development 76 1094-1105. (doi:10.1002/mrd. 21075)

Slattery SD, Mancini MA, Brinkley BR \& Hall RM 2009 Aurora-C kinase supports mitotic progression in the absence of Aurora-B. Cell Cycle $\mathbf{8}$ 2984-2994.

Solc P, Saskova A, Baran V, Kubelka M, Schultz RM \& Motlik J 2008 CDC25A phosphatase controls meiosis I progression in mouse oocytes. Developmental Biology 317 260-269. (doi:10.1016/j.ydbio. 2008.02.028)

Steigemann P, Wurzenberger C, Schmitz MH, Held M, Guizetti J, Maar S \& Gerlich DW 2009 Aurora B-mediated abscission checkpoint protects against tetraploidization. Cell 136 473-484. (doi:10.1016/j.cell.2008. 12.020)

Sutcliffe MJ, Darling SM \& Burgoyne PS 1991 Spermatogenesis in XY, XYSxra and XOSxra mice: a quantitative analysis of spermatogenesis throughout puberty. Molecular Reproduction and Development 30 81-89. (doi:10.1002/mrd.1080300202)
Swain JE, Ding J, Wu J \& Smith GD 2008 Regulation of spindle and chromatin dynamics during early and late stages of oocyte maturation by aurora kinases. Molecular Human Reproduction 14 291-299. (doi:10. 1093/molehr/gan015)

Terret ME, Wassmann K, Waizenegger I, Maro B, Peters JM \& Verlhac MH 2003 The meiosis I-to-meiosis II transition in mouse oocytes requires separase activity. Current Biology 13 1797-1802. (doi:10.1016/j.cub. 2003.09.032)

Tsafriri A, Chun SY, Zhang R, Hsueh AJ \& Conti M 1996 Oocyte maturation involves compartmentalization and opposing changes of cAMP levels in follicular somatic and germ cells: studies using selective phosphodiesterase inhibitors. Developmental Biology 178 393-402. (doi:10.1006/ dbio.1996.0226)

Tseng TC, Chen SH, Hsu YP \& Tang TK 1998 Protein kinase profile of sperm and eggs: cloning and characterization of two novel testis-specific protein kinases (AIE1, AIE2) related to yeast and fly chromosome segregation regulators. DNA and Cell Biology 17 823-833. (doi:10. 1089/dna.1998.17.823)

Tsurumi C, Hoffmann S, Geley S, Graeser R \& Polanski Z 2004 The spindle assembly checkpoint is not essential for CSF arrest of mouse oocytes. Journal of Cell Biology 167 1037-1050. (doi:10.1083/jcb.200405165)

Uzbekova S, Arlot-Bonnemains Y, Dupont J, Dalbies-Tran R, Papillier P, Pennetier S, Thelie A, Perreau C, Mermillod P, Prigent C et al. 2008 Spatio-temporal expression patterns of aurora kinases A, B, and C and cytoplasmic polyadenylation-element-binding protein in bovine oocytes during meiotic maturation. Biology of Reproduction 78 218-233. (doi:10.1095/biolreprod.107.061036)

Vogt E, Kipp A \& Eichenlaub-Ritter U 2009 Aurora kinase B, epigenetic state of centromeric heterochromatin and chiasma resolution in oocytes. Reproductive Biomedicine Online 19 352-368. (doi:10.1016/S14726483(10)60169-1)

Wassmann K, Niault T \& Maro B 2003 Metaphase I arrest upon activation of the Mad2-dependent spindle checkpoint in mouse oocytes. Current Biology 13 1596-1608. (doi:10.1016/j.cub.2003.08.052)

Weaver BA, Silk AD \& Cleveland DW 2006 Cell biology: nondisjunction, aneuploidy and tetraploidy. Nature 442 E9-E10. (doi:10.1038/nature 05139)

Yang KT, Li SK, Chang CC, Tang CJ, Lin YN, Lee SC \& Tang TK 2010 Aurora $\mathrm{C}$ kinase deficiency causes cytokinesis failure in meiosis I and production of large polyploid oocytes in mouse. Molecular Biology of the Cell 21 2371-2383. (doi:10.1091/mbc.E10-02-0170)

Yin S, Wang Q, Liu JH, Ai JS, Liang CG, Hou Y, Chen DY, Schatten H \& Sun QY 2006 Bub1 prevents chromosome misalignment and precocious anaphase during mouse oocyte meiosis. Cell Cycle 5 2130-2137.

Received 12 May 2010

First decision 16 June 2010

Accepted 21 July 2010 